\title{
2 Bisherige Forschungsergebnisse und Erklärungsversuche
}

Die Forschung zu Schul-Amokläufen ist noch relativ jung. Erkenntnisse entstanden aus einer Ex-Post-Betrachtung und durch retrospektive Analysen. Die Ursachenforschung gestaltet sich deshalb schwierig, weil die Häufigkeit von Schul-Amokläufen relativ niedrig ist und die Täter nur in wenigen Fällen nach ihrer Tat befragt werden konnten, denn über 90\% der Täter töteten sich nach begangener Tat selbst, was das Wissen über die vorangegangene Dynamik des Schul-Amoklaufs einschränkt. Außerdem wiesen die ersten Täter nur wenige Gemeinsamkeiten auf, sodass weder ein eindeutiges Profil noch ein zwingender Zusammenhang zwischen den sozialen Beziehungen der Täter und ihrer Bereitschaft zur Gewaltanwendung entwickelt werden konnte.

Andererseits zeigt sich in der Öffentlichkeit und bei allen, die mit diesen unfassbaren Taten konfrontiert werden, sehr schnell ein hohes Erklärungsbedürfnis. Man meint, es müsse doch Gründe für eine solche Tat geben, auf deren Grundlage schnelle Lösungsmöglichkeiten entwickelt werden könnten. Man geht davon aus, dass Taten wie Schul-Amok und School Shooting - ebenso wie suizidales Verhalten oder Affekthandlungen - ein spezifisches Muster zeigen sollten, ähnlich den „Komplexreaktionen“ (Adler 2010) der klassischen Psychoanalyse. 


\subsection{Frühe Feststellungen zum Phänomen Schul-Amok und School Shooting}

Experten des National Research Council in den USA sowie deutsche Experten wie Hoffmann et al. (2009) und Robertz (2004) versuchten, die Beweggründe der jugendlichen Schul-Amokläufer zu verstehen. Hoffmann et al. (2009) und Robertz (2004, 2007) fanden wichtige Warnsignale für ein School shooting und erarbeiteten entsprechende Gegenmaßnahmen sowie Richtlinien für den Ernstfall. Sie vermitteln diese in Fortbildungen an Multiplikatoren wie Lehrer, Schulpsychologische Dienste, Ärzte und Therapeuten, Polizisten, Mitarbeitern von Unfallkassen.

Der National Research Council in den USA veröffentlichte schon Mitte 2002 einen Report über Schulmassaker, in dem Psychologen, Kriminologen und Verhaltensforscher Tatabläufe und Täterpersönlichkeiten analysieren und Wege zur Prävention aufzuzeigen versuchen.

Folgende Gemeinsamkeiten konnten festgestellt werden:

- Alle Täter waren Jungen.

- Fast alle hatten ungehinderten Zugang zu Waffen.

- Die meisten stammten aus weißen Mittelschichtfamilien.

- Die Täter stammten aus ,funktionierenden“ Familien und nicht aus zerrütteten Familienverhältnissen.

- Allen Tätern gemeinsam war eine ausgeprägte Bereitschaft zum Selbstmord, die sie $\mathrm{zu} 78 \%$ vorher artikulierten.

- Die Täter wiesen in überdurchschnittlichem Maß Anzeichen psychiatrischer Erkrankungen auf, meist Depressionen oder Schizophrenie.

- $98 \%$ der Täter hatten im unmittelbaren Vorfeld der Tat einen signifikanten Verlust oder eine Niederlage erlitten.

- $71 \%$ hatten das Gefühl, ausgegrenzt oder gemobbt worden zu sein. Diagnostiziert wurden diese Veränderungen jedoch grundsätzlich erst nach der Tat.

- In $81 \%$ der Schul-Amoktaten hatte der Täter zuvor mindestens eine Person in sein Vorhaben eingeweiht.

- Gewaltverherrlichende Video- und Computerspiele sowie Gewalt-glorifizierende Musik spielten im Leben der meisten Attentäter zwar eine gewisse, aber nicht eine dominante Rolle.

- 59\% der Täter hatten einen intensiven Konsum von Gewaltmedien.

- Ein Drittel der Täter stirbt.

- Überlebende geben Amnesien an oder sind in prozesstaktische Überlegungen verstrickt.

- Schul-Amoktäter reißen nicht nur andere in den Tod, sondern nehmen oft ihr eigenes Ableben in Kauf (Wuketits 2010). 
Die ersten US-amerikanischen Studien machen deutlich, dass es sich bei den Tätern um introvertierte Jugendliche handelte, die relativ geringe, schwache soziale Bindungen hatten und für ihr weiteres Leben kaum eine Zukunftsperspektive sahen. Oft hatten sie in den Monaten oder Jahren vor ihrer Tat schwere persönliche Kränkungen erlitten, wurden etwa von der Schule verwiesen, fühlten sich von Lehrern unter Druck gesetzt oder von den Mitschülern abgelehnt und schikaniert. Vor ihrem Amoklauf hatten sie meist intensive Erfahrungen mit Schusswaffen gesammelt und ihre Tat über einen längeren Zeitraum hinweg genauestens vorbereitet.

Auch Bondü und Scheithauer (2009) stellen fest, dass die meisten Schul-Amoktäter und School Shooter Probleme mit Verlusterlebnissen wie Schulabbrüchen oder Schulausschlüssen hatten oder erhebliche Schwierigkeiten besaßen, mit persönlichem Versagen fertigzuwerden. Viele entwickelten schon im Vorfeld ihrer Tat Suizidideen oder unternahmen einen Suizidversuch. Meistens gab es Warnsignale, die den Taten vorausgingen.

Es ist wichtig, eine „Kultur des Hinschauens“ zu entwickeln. 